\title{
10-Hydroxy-2-decenoic Acid, the Major Lipid Component of Royal Jelly, Extends the Lifespan of Caenorhabditis elegans through Dietary Restriction and Target of Rapamycin Signaling
}

\author{
Yoko Honda, ${ }^{1}$ Yoko Araki, ${ }^{2}$ Taketoshi Hata, ${ }^{2}$ Kenji Ichihara, ${ }^{2}$ Masafumi Ito, ${ }^{3}$ \\ Masashi Tanaka, ${ }^{1}$ and Shuji Honda ${ }^{1}$ \\ ${ }^{1}$ Department of Genomics for Longevity and Health, Tokyo Metropolitan Institute of Gerontology, Sakaecho, Itabashiku, \\ Tokyo 173-0015, Japan \\ ${ }^{2}$ Nagaragawa Research Center, Api Company Limited, Nagara, Gifu 502-0071, Japan \\ ${ }^{3}$ Research Team for Mechanism of Aging, Tokyo Metropolitan Institute of Gerontology, Sakaecho, Itabashiku, Tokyo 173-0015, Japan \\ Correspondence should be addressed to Shuji Honda; hondas@center.tmig.or.jp
}

Received 11 November 2014; Revised 23 January 2015; Accepted 23 January 2015

Academic Editor: F. R. Ferraro

Copyright (C) 2015 Yoko Honda et al. This is an open access article distributed under the Creative Commons Attribution License, which permits unrestricted use, distribution, and reproduction in any medium, provided the original work is properly cited.

\begin{abstract}
Royal jelly (RJ) produced by honeybees has been reported to possess diverse health-beneficial properties and has been implicated to have a function in longevity across diverse species as well as honeybees. 10-Hydroxy-2-decenoic acid (10-HDA), the major lipid component of RJ produced by honeybees, was previously shown to increase the lifespan of Caenorhabditis elegans. The objective of this study is to elucidate signaling pathways that are involved in the lifespan extension by 10-HDA. 10-HDA further extended the lifespan of the daf-2 mutants, which exhibit long lifespan through reducing insulin-like signaling (ILS), indicating that 10-HDA extended lifespan independently of ILS. On the other hand, 10-HDA did not extend the lifespan of the eat-2 mutants, which show long lifespan through dietary restriction caused by a food-intake defect. This finding indicates that 10-HDA extends lifespan through dietary restriction signaling. We further found that 10-HDA did not extend the lifespan of the long-lived mutants in daf-15, which encodes Raptor, a target of rapamycin (TOR) components, indicating that 10-HDA shared some longevity control mechanisms with TOR signaling. Additionally, 10-HDA was found to confer tolerance against thermal and oxidative stress. 10-HDA increases longevity not through ILS but through dietary restriction and TOR signaling in C. elegans.
\end{abstract}

\section{Introduction}

One of the most important challenges in the study of aging is the discovery of compounds with longevity-promoting activities and the elucidation of their underlying mechanisms. Such compounds could provide potential nutraceutical or pharmaceutical approaches to slow aging and the onset of age-related diseases in humans [1]. Royal jelly (RJ) is produced by the hypopharyngeal, postcerebral, and mandibular glands of the worker bees and has been implicated to be involved in the longer lifespan of queens in contrast with workers in the honeybee Apis mellifera L., because queens are fed throughout their lives with RJ, whereas workers are fed RJ for only a short period of time during their larval stage [2-5]. RJ has been also reported to extend the lifespan of nematodes [6, 7], flies [8,9], and mice [10] indicating that RJ has a common role in longevity across phyla. RJ comprises proteins, sugars, lipids, vitamins, and free amino acids, together with a variety of bioactive substances [11]. The identities of the components that play critical roles in longevity are not fully understood. A single protein, termed royalactin contained in $\mathrm{RJ}$, was reported to extend the lifespan of D. melanogaster and C. elegans $[9,12]$. We previously found 10-hydroxy-2-decenoic acid (10-HDA), which is the major lipid component of RJ [13] and has several health-beneficial effects in mammals [14], such as antitumor activity [15], antiinflammatory activity [16], and antiangiogenic activity [17]. It also extends the lifespan of C. elegans [6]. However, how $10-$ HDA extends lifespan is not well elucidated. 
It has been reported that lifespan is regulated mainly through insulin-like signaling (ILS) and dietary restriction signaling in C. elegans as well as Drosophila melanogaster and other experimental animals [18]. In the present study, we found that 10-HDA extended the lifespan of $C$. elegans not through ILS but through dietary restriction signaling. Dietary restriction signaling has been reported to mediate lifespan extension through various downstream signaling pathways including target of rapamycin (TOR) signaling [19]. We suggest in this report that 10-HDA extends the lifespan via the TOR signaling.

\section{Methods}

2.1. Culture and Strains of C. elegans. C. elegans strains were maintained at $20^{\circ} \mathrm{C}$ on nematode growth medium agar with Escherichia coli OP50 as a food source, as previously described [20]. The N2 Bristol strain was used as the wild type $C$. elegans. The mutant strains used in this study were CB1370: daf-2(e1370) III; DA465: eat-2 (ad465) II; CB138: unc24(e138) IV; DR412: unc-24(e138)/daf-15(m81) IV; and LG344: geIs8[gpa-4p::skn-lb::gfp + rol-6(su1006)].

2.2. Treatment with 10-HAD. 10-HDA was purchased from Alfresa Pharma Co., Ltd., Osaka, Japan. 10-HDA was added to liquid NGM that had been autoclaved and cooled to $50^{\circ} \mathrm{C}$, using DMSO as a solvent at a final concentration of $0.03 \%$. The media were immediately dispensed into Petri dishes. 10-HDA was provided at $20^{\circ} \mathrm{C}$ from adult 0 -day to death in the lifespan assay and from hatched L1 stage in the progeny production assay. Experiments involving 10-HDA were conducted in parallel with those involving a control group treated with an equivalent volume of DMSO.

2.3. Lifespan Determination. Lifespan was determined as previously described [6]. UV-killed E. coli strain OP50 was used as a food source in the experiment to avoid any effects of live E. coli on 10-HDA and any effects of 10-HDA on growth and metabolism of live E. coli. Lifespan under UVkilled E. coli is longer compared to that under live E. coli probably because of the diminished toxicity of growing bacteria [21]. Worms were raised until the L4 molt and were then transferred onto a new plate containing $40 \mu \mathrm{M} 5$-fluoro$2^{\prime}$-deoxyuridine (Sigma Aldrich, St. Louis, MO, USA) to prevent self-fertilization $[22,23]$. The day of transfer at the L4 molt was counted as adult 0 -day. Worms were judged to be dead when they did not respond to a mechanical stimulus. To focus on aging, worms that had become desiccated on the side of the plate after crawling off or those that displayed extruded internal organs were excluded from our analysis. The results of the survival assays were analyzed using the Kaplan-Meier method, and significance was measured with the log-rank test using the statistical analysis package StatMate III (ATMS, Tokyo, Japan).

2.4. Measurement of Progeny Production. Single newly hatched N2 worm was placed on a plate containing UV-killed OP50. Worms were transferred every day or every other day

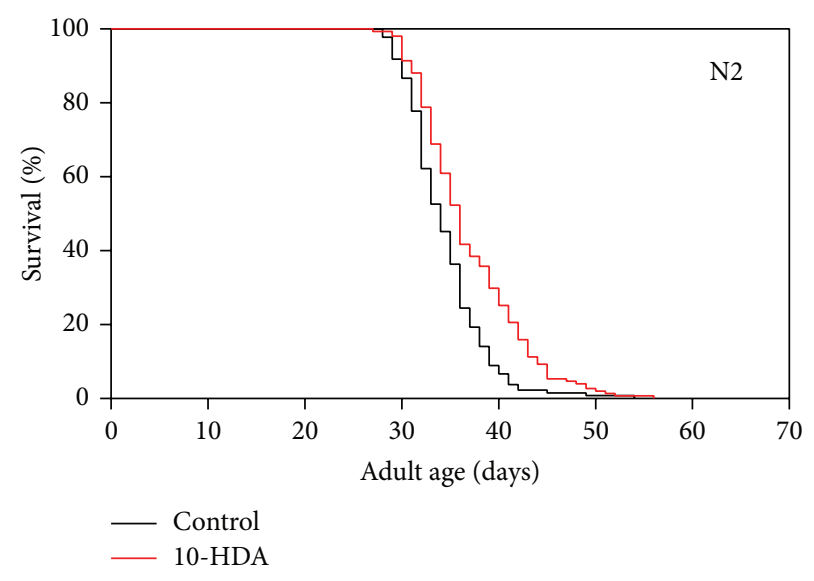

Figure 1: The effect of 10-HDA on the lifespan of N2 wild type $C$. elegans. The curves shown in Figures 1, 2, 3, and 5 represent pooled data from three to four experiments (Table 1).

to fresh plates. The resulting progenies were left to develop for 2 days for measurement of progeny number.

2.5. Assays of Stress Resistance. To assess thermotolerance, young adult hermaphrodites were placed on NGM plates at $35^{\circ} \mathrm{C}$ and scored for viability. To assess oxidative stress, young adult hermaphrodites were placed on NGM plates, which included $50 \mathrm{mM}$ paraquat (Sigma Aldrich) at $20^{\circ} \mathrm{C}$ and were scored for viability.

\section{Results}

3.1. 10-HDA Extends Lifespan Independently of ILS. As shown in Figure 1 and Table 1, 10-HDA extended the lifespan of N2, a wild type strain of C. elegans as previously described [6]. To investigate whether this lifespan extension effect of 10HDA was due to ILS, we evaluated the effect of 10-HDA on the lifespan of the insulin-like receptor daf-2 mutants, which reduce ILS and show long lifespan [18]. We found that 10HDA further extended the lifespan of daf-2 (Figure 2 and Table 1) indicating that 10-HDA affected lifespan independently of ILS. The previous finding that 10-HDA extended the lifespan of the mutants in daf-16, which encodes the FOXO transcription factor, the downstream target of ILS [6], also supports this notion. Alternatively, 10-HDA appeared to extend the lifespan of the daf-2 mutants to a greater extent than that of the wild type (Figure 2 and Table 1) suggesting some interaction between 10-HDA and ILS.

3.2. 10-HDA Extends Lifespan through Dietary Restriction Signaling. To determine whether the lifespan extension by 10 -HDA is mediated through dietary restriction signaling, we examined the effect of 10-HDA on the lifespan of the eat-2 mutants, which display the extended lifespan through the feeding impairment-based dietary restriction [24]. As shown in Figure 3 and Table 1,10-HDA did not further extend the lifespan of the eat-2 mutants, suggesting that 10-HDA shared common lifespan control mechanisms with dietary restriction signaling. We wondered if 10-HDA directly exerts 
TABLE 1: The effects of 10-HDA on the lifespan of various mutants.

\begin{tabular}{|c|c|c|c|c|c|c|c|c|}
\hline Exp. & Genotype & Treatment & Mean life span \pm SEM (days) & $\%$ change & Maximum life span (days) & $\%$ change & $N$ (animal) & Significance \\
\hline \multirow{2}{*}{1} & $\mathrm{~N} 2$ & Control & $34.8 \pm 0.8$ & \multirow{2}{*}{+11} & 54 & \multirow{2}{*}{-4} & 34 & \multirow{2}{*}{$P<0.01$} \\
\hline & $\mathrm{N} 2$ & 10-HDA $25 \mu \mathrm{M}$ & $38.7 \pm 1.0$ & & 52 & & 26 & \\
\hline \multirow{2}{*}{2} & $\mathrm{~N} 2$ & Control & $34.4 \pm 0.6$ & \multirow{2}{*}{+10} & 49 & \multirow{2}{*}{+14} & 45 & \multirow{2}{*}{$P<0.01$} \\
\hline & $\mathrm{N} 2$ & 10-HDA $25 \mu \mathrm{M}$ & $37.7 \pm 0.8$ & & 56 & & 29 & \\
\hline \multirow{2}{*}{3} & N2 & Control & $34.3 \pm 0.5$ & \multirow{2}{*}{+6} & 45 & \multirow{2}{*}{+11} & 56 & \multirow{2}{*}{$P<0.01$} \\
\hline & $\mathrm{N} 2$ & 10-HDA $25 \mu \mathrm{M}$ & $36.2 \pm 0.5$ & & 50 & & 95 & \\
\hline \multirow{2}{*}{1} & $d a f-2$ & Control & $61.3 \pm 12.3$ & \multirow{2}{*}{+10} & 82 & \multirow{2}{*}{+20} & 31 & \multirow{2}{*}{$P<0.01$} \\
\hline & daf-2 & 10-HDA $25 \mu \mathrm{M}$ & $67.2 \pm 11.7$ & & 98 & & 34 & \\
\hline \multirow{2}{*}{2} & daf-2 & Control & $63.7 \pm 10.5$ & \multirow{2}{*}{+24} & 87 & \multirow{2}{*}{+46} & 49 & \multirow{2}{*}{$P<0.01$} \\
\hline & daf-2 & 10-HDA $25 \mu \mathrm{M}$ & $79.2 \pm 17.5$ & & 127 & & 41 & \\
\hline \multirow{2}{*}{3} & daf-2 & Control & $63.0 \pm 11.9$ & \multirow{2}{*}{+14} & 83 & \multirow{2}{*}{+18} & 53 & \multirow{2}{*}{$P<0.01$} \\
\hline & $d a f-2$ & 10-HDA $25 \mu \mathrm{M}$ & $71.8 \pm 13.9$ & & 98 & & 61 & \\
\hline \multirow{2}{*}{1} & eat-2 & Control & $32.5 \pm 2.8$ & \multirow{2}{*}{+6} & 39 & \multirow{2}{*}{+25} & 23 & \\
\hline & eat-2 & 10-HDA $25 \mu \mathrm{M}$ & $34.5 \pm 4.4$ & & 49 & & 21 & \\
\hline 2 & eat-2 & Control & $39.8 \pm 4.8$ & -13 & 56 & -14 & 30 & \\
\hline 2 & eat -2 & 10-HDA $25 \mu \mathrm{M}$ & $34.8 \pm 5.1$ & -13 & 48 & -14 & 11 & \\
\hline 3 & eat-2 & Control & $37.0 \pm 5.3$ & -7 & 54 & -4 & 71 & \\
\hline 3 & eat-2 & 10-HDA $25 \mu \mathrm{M}$ & $34.4 \pm 4.4$ & -1 & 52 & -4 & 83 & \\
\hline & unc-24/+ & Control & $37.8 \pm 0.8$ & +4 & 46 & +9 & 58 & \\
\hline 1 & unc-24/+ & 10-HDA $25 \mu \mathrm{M}$ & $40.2 \pm 1.1$ & +4 & 50 & +9 & 61 & \\
\hline & unc-24/daf-15 & Control & $39.6 \pm 1.3$ & -5 & 49 & -14 & 71 & \\
\hline & unc-24/daf-15 & 10-HDA $25 \mu \mathrm{M}$ & $36.4 \pm 0.6$ & -5 & 42 & -14 & 65 & \\
\hline & unc-24/+ & Control & $36.6 \pm 0.5$ & +4 & 46 & +9 & 58 & \\
\hline 2 & unc-24/+ & 10-HDA $25 \mu \mathrm{M}$ & $38.1 \pm 0.6$ & +4 & 50 & & 61 & $P<0.01$ \\
\hline 2 & unc-24/daf-15 & Control & $37.7 \pm 0.5$ & -5 & 49 & -14 & 71 & $P<0.01$ \\
\hline & unc-24/daf-15 & 10-HDA $25 \mu \mathrm{M}$ & $35.8 \pm 0.4$ & -5 & 42 & -14 & 65 & \\
\hline & $u n c-24 /+$ & Control & $37.7 \pm 0.6$ & +6 & 47 & +13 & 43 & \\
\hline 3 & unc-24/+ & 10-HDA $25 \mu \mathrm{M}$ & $39.8 \pm 0.7$ & +6 & 53 & & 47 & $P<0.01$ \\
\hline & unc-24/daf-15 & Control & $39.1 \pm 0.6$ & -5 & 53 & -11 & 85 & \\
\hline & unc-24/daf-15 & 10-HDA $25 \mu \mathrm{M}$ & $37.2 \pm 0.4$ & -5 & 47 & -11 & 83 & \\
\hline & unc-24/+ & Control & $36.5 \pm 4.6$ & +7 & 46 & +11 & 38 & \\
\hline 4 & unc-24/+ & 10-HDA $25 \mu \mathrm{M}$ & $39.0 \pm 5.8$ & & 51 & +11 & 41 & $P<0.01$ \\
\hline 4 & unc-24/daf-15 & Control & $37.8 \pm 5.3$ & -3 & 50 & & 37 & $P<0.01$ \\
\hline & unc-24/daf-15 & 10-HDA $25 \mu \mathrm{M}$ & $36.5 \pm 4.1$ & -3 & 45 & & 39 & \\
\hline
\end{tabular}

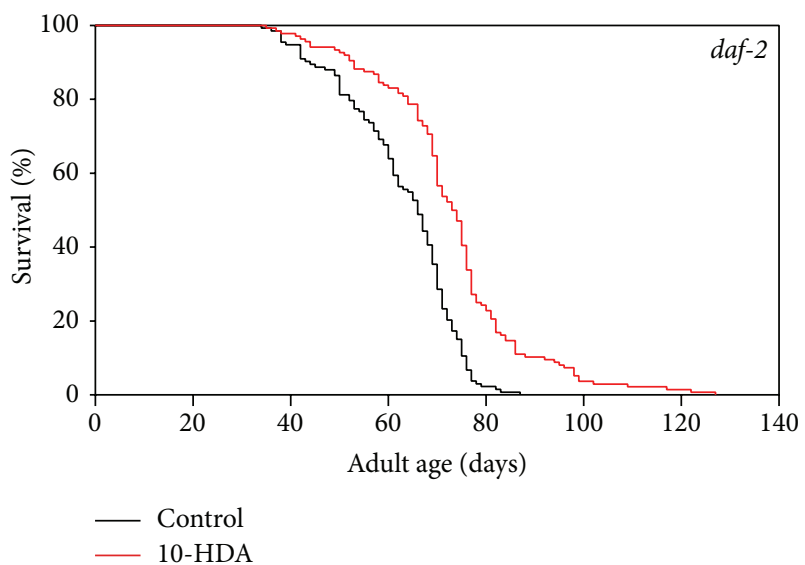

FIGURE 2: The effect of 10-HDA on the lifespan of the daf- 2 mutants.

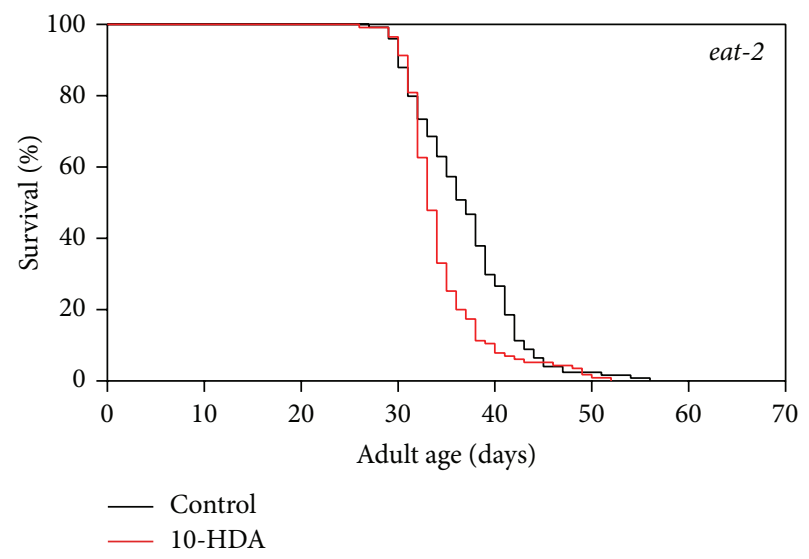

Figure 3: The effect of 10-HDA on the lifespan of the eat-2 mutants. 


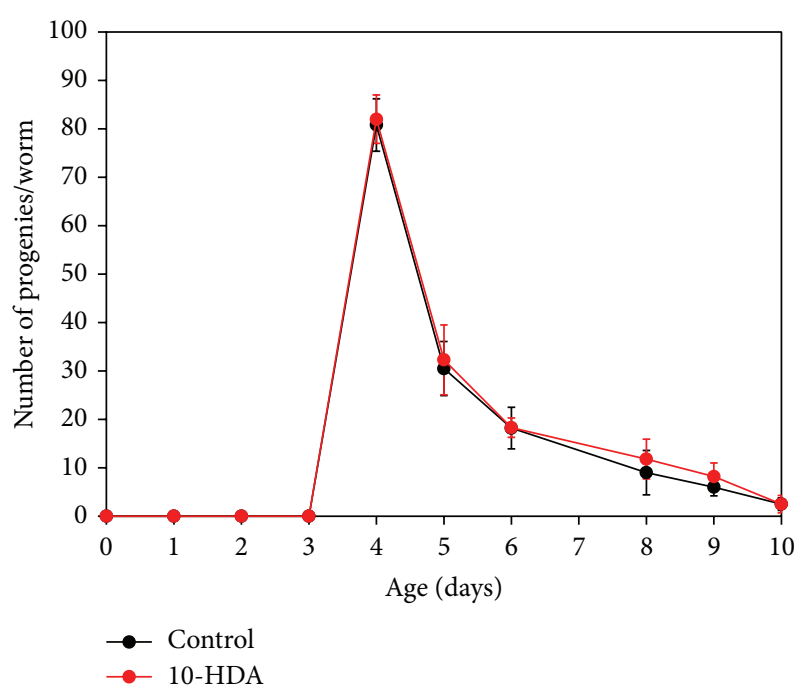

FIGURE 4: The effect of 10-HDA on the progeny production of wild type $C$. elegans. The progeny production is plotted against age at transfer. Mean values and SD are shown $(n=6)$. Day 0 corresponds to the hatched L1 stage.

dietary restriction. Dietary-restricted worms are known to produce progeny in a delayed manner [17]. 10-HDA-treated worms produced progeny in a similar manner to untreated ones (Figure 4), suggesting that 10-HDA is unlikely to directly restrict diet. Taken together, these findings suggest that 10HDA extended lifespan by affecting the downstream process of the dietary restriction signaling.

3.3. 10-HDA Extends Lifespan through TOR Signaling. Dietary restriction has been reported to extend lifespan through various downstream signalings including TOR signaling [15]. To determine whether 10-HDA extended lifespan via the TOR signaling, we evaluated the effect of 10-HDA on the lifespan of mutants in daf-15, which encodes Raptor (the regulatory associated protein of mTOR), a component of the TOR complex [25]. The daf-15 homozygous mutants show larval arrest and heterozygotes display lifespan extension [22]. As shown in Figure 5(b) and Table 1, 10-OHDA did not further extend the lifespan of the daf-15 heterozygous mutants; 10HDA extended the lifespan of the control unc-24/+ mutants (Figure 5(a) and Table 1). This finding suggests that lifespan extension by $10-\mathrm{HDA}$ is mediated through TOR signaling.

\subsection{0-HDA Confers Tolerance against Thermal and Oxidative} Stresses. To assess whether 10-HDA confers tolerance against thermal and oxidative stresses, we examined the effects of 10-HDA treatment on survival during heat exposure and paraquat exposure. As shown in Figures 6(a) and 6(b), 10HDA increased survival during both heat exposure and paraquat exposure.

\section{Discussion}

The findings we have presented here suggest that 10-HDA, the major lipid component of RJ, extends the lifespan of C. elegans through dietary restriction and TOR signaling. RJ is believed to have several components with lifespan-extending activity, including peptides $(6)$, royalactin $(9,12)$, and 10-HDA, which work through a variety of signaling pathways, such as ILS, EGF, or TOR. This seems to indicate that RJ originally has diverse functions in honeybees, such as cast differentiation, queen longevity, and nourishment.

A variety of compounds have been shown to extend the lifespan of C. elegans [1]. Among them, anticonvulsants like ethosuximide [26], valproic acid [27], icariin [28], and caffeic acid phenethyl ester [29] extend lifespan via ILS. The present study showed that 10-HDA further extended the lifespan of daf-2 mutants that showed long lifespan via ILS, indicating that 10-HDA extends lifespan independently of ILS.

On the other hand, dietary restriction mimetics including 2-deoxyglucose extend lifespan like direct dietary restriction [30]. Further, several compounds that target downstream of dietary restriction signaling also extend lifespan: $\alpha$ ketoglutarate via the TOR signaling [31]; diallyl trisulfide [32] via SKN-1 pathway; metformin via AMP-activated protein kinase (AMPK); and SKN-1 pathways [33]. The SKN1 transcription factor is the ortholog of mammalian Nrf2 and known to control stress protection. 10-HDA was found to confer tolerance against thermal and oxidative stresses (Figures 6(a) and 6(b)). However, we failed to find the induction of $s k n-1$ by 10-HDA treatment using the reporter of skn-1: geIs8 [gpa-4p::skn-1b::gfp] (unpublished observation), suggesting that 10-HDA may not be involved in the SKN-1 pathway. Metformin has also been reported to retard aging by directly affecting metabolism of folate and methionine in E. coli, the worm's food [34]. Resveratrol extended lifespan via Sir2 [35] and AMPK pathways [19]. However, resveratrol has also been reported not to extend the lifespan of worms or flies [36] or mice [37]. Furthermore, oxaloacetate extended worm lifespan via both IIS and dietary restriction [38] but was reported not to extend mouse lifespan [37].

In the present study, we found that 10-HDA extended C. elegans lifespan via dietary restriction and the TOR pathway. RJ, whose major lipid component is 10-HDA, plays an important role in an epigenetic fate determination between worker and long-lived queen honeybees with identical genome composition $[2-5,39]$. TOR has been reported to participate in the caste fate determination in honeybees [4042]. Although it is unknown whether 10-HDA is involved in the caste fate determination, it may be relevant that 10 HDA functions as an inhibitor of histone deacetylase, which is known to play a main role in epigenesis [43] and that 10-HDA affects the expression of histone deacetylase 3 and DNA methyltransferase 3 , both of which also play a role in epigenesis in honeybees [44].

TOR is known to be a nutrient sensor and controls lifespan as well as protein synthesis and degradation, cell growth, and autophagy [45-48]. Rapamycin, which inhibits TOR, extends the lifespan of yeasts [49], nematodes [50], flies [51], and mice [52]. However, chronic administration of rapamycin in rodents has several side effects such as glucose intolerance, insulin tolerance, and cataracts [53-57]. It may be possible that 10 -HDA contained in RJ which is widely taken as a health food could be used as nutraceutical intervention 


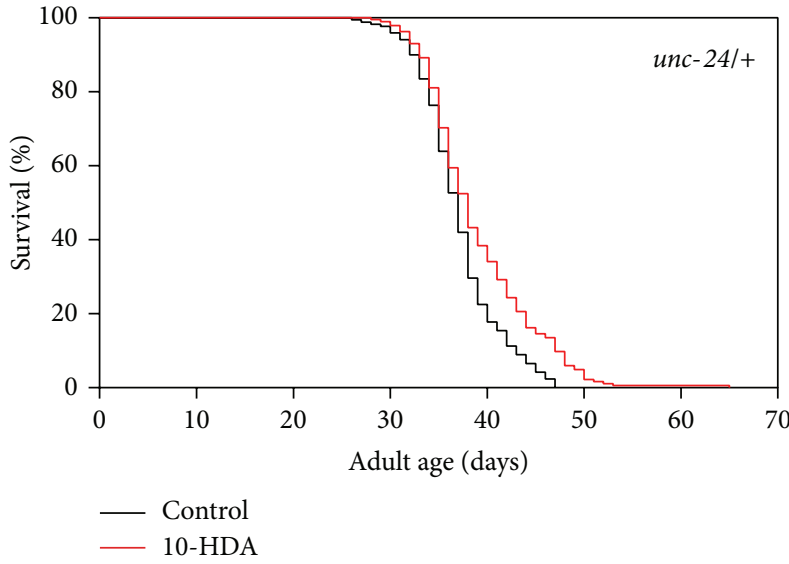

(a)

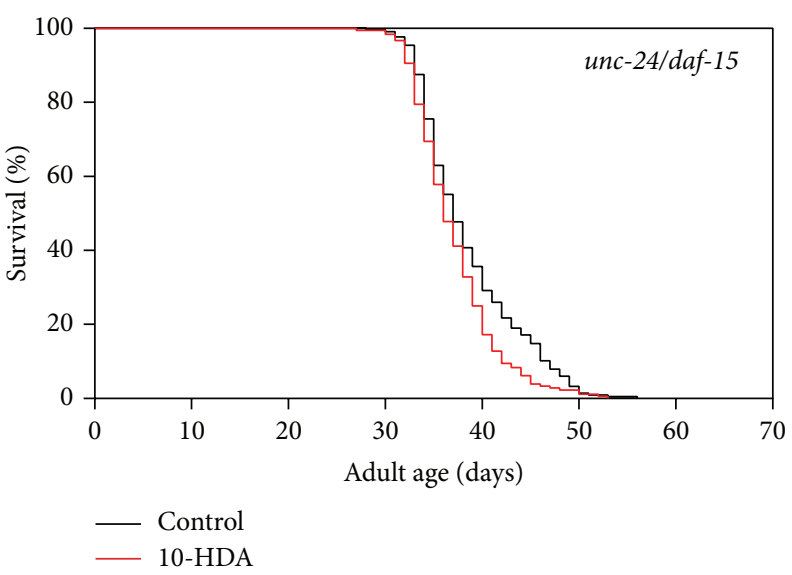

(b)

FIGURE 5: The effect of 10-HDA on the lifespan of the daf-15 mutants. (a) unc-24/+ and (b) unc-24/daf-15.

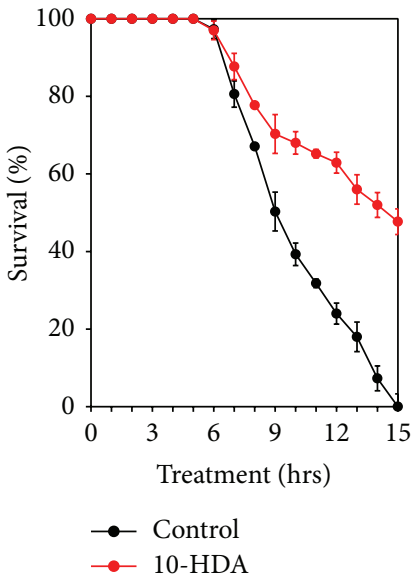

(a)

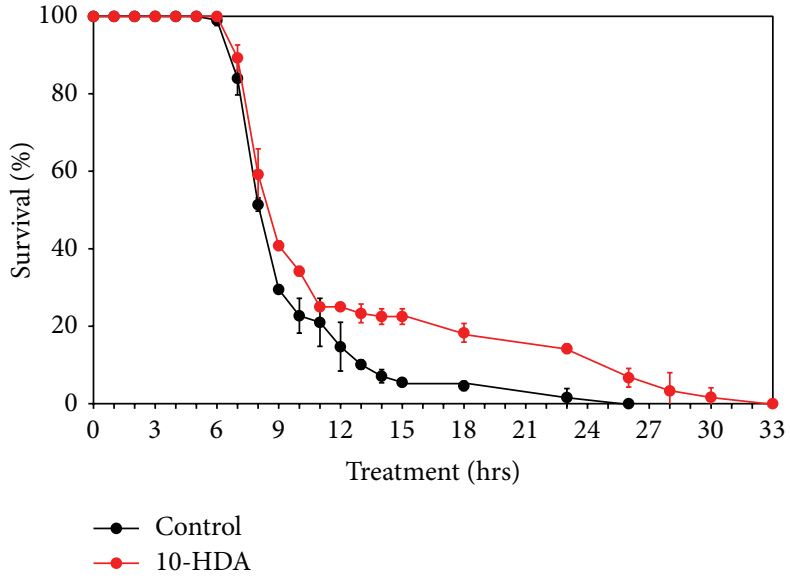

(b)

Figure 6: (a) Thermal stress. (b) Oxidative stress. Treatment with or without $25 \mu \mathrm{M} 10$-HDA began at the egg stage. Mean values and SD of triplicate samples are plotted.

aimed at mammalian TOR (mTOR) inhibition to delay aging and the onset of age-related diseases.

\section{Conclusions}

The present study indicates that 10-HDA extends lifespan of C. elegans not via ILS but via dietary restriction and the TOR signaling. It may be possible that 10-HDA contained in RJ which is widely taken by human as a health food could be used as nutraceutical intervention aimed at mTOR inhibition to delay aging and the onset of age-related diseases.

\section{Conflict of Interests}

This work was essentially supported by the Tokyo Metropolitan Institute of Gerontology financially and was partially funded by Api Company Limited, which engages in the manufacture and sale of royal jelly products and of which Yoko
Araki, Taketoshi Hata, and Kenji Ichihara are employees. The authors confirm that this declaration does not alter their adherence to all the Journal of Aging Research policies on sharing data.

\section{Acknowledgments}

Some strains were provided by the CGC, which is funded by NIH Office of Research Infrastructure Programs (P40 OD010440).

\section{References}

[1] M. Lucanic, G. J. Lithgow, and S. Alavez, "Pharmacological lifespan extension of invertebrates," Ageing Research Reviews, vol. 12, no. 1, pp. 445-458, 2013.

[2] M. L. Winston, The Biology of the Honey Bee, Harvard University Press, Cambridge, Mass, USA, 1991. 
[3] S. C. Remolina and K. A. Hughes, "Evolution and mechanisms of long life and high fertility in queen honey bees," Age, vol. 30, no. 2-3, pp. 177-185, 2008.

[4] A. Chittka and L. Chittka, "Epigenetics of royalty," PLoS Biology, vol. 8, no. 11, Article ID e1000532, 2010.

[5] R. E. Page Jr. and C. Y. S. Peng, "Aging and development in social insects with emphasis on the honey bee, Apis mellifera L," Experimental Gerontology, vol. 36, no. 4-6, pp. 695-711, 2001.

[6] Y. Honda, Y. Fujita, H. Maruyama et al., "Lifespan-extending effects of royal jelly and its related substances on the nematode Caenorhabditis elegans," PLoS ONE, vol. 6, no. 8, Article ID e23527, 2011.

[7] X. Wang, L. F. Cook, L. M. Grasso, M. Cao, and Y. Dong, "Royal jelly-mediated prolongevity and stress resistance in Caenorhabditis elegans is possibly modulated by the interplays of DAF-16, SIR-2.1, HCF-1, and 14-3-3 proteins," The Journal of Gerontology, A Biological Science and Medical Science, 2014.

[8] T. S. Gardner, "The use of Drosophila melanogaster as a screening agent for longevity factors; pantothenic acid as a longevity factor in royal jelly," The Journals of Gerontology, vol. 3, no. 1, pp. $1-8,1948$.

[9] M. Kamakura, "Royalactin induces queen differentiation in honeybees," Nature, vol. 473, no. 7348, pp. 478-483, 2011.

[10] S. I. Inoue, S. Koya-Miyata, S. Ushio, K. Iwaki, M. Ikeda, and M. Kurimoto, "Royal Jelly prolongs the life span of $\mathrm{C} 3 \mathrm{H} / \mathrm{HeJ}$ mice: correlation with reduced DNA damage," Experimental Gerontology, vol. 38, no. 9, pp. 965-969, 2003.

[11] T. Takenaka, "Chemical composition of royal jelly," Honeybee Science, vol. 3, no. 2, pp. 69-74, 1982.

[12] G. Detienne, W. de Haes, U. R. Ernst, L. Schoofs, and L. Temmerman, "Royalactin extends lifespan of Caenorhabditis elegans through epidermal growth factor signaling," Experimental Gerontology, vol. 60, pp. 129-135, 2014.

[13] A. Butenandt and H. Rembold, "Royal jelly of the honeybee. I. Isolation, constitution analysis, and incidence of 10-hydroxydelta 2-decenoic acid," Hoppe-Seyler's Zeitschrift für Physiologische Chemie, vol. 203, no. 5-6, pp. 284-289, 1957.

[14] X. Li, C. Huang, and Y. Xue, "Contribution of lipids in honeybee (Apis mellifera) royal jelly to health," Journal of Medicinal Food, vol. 16, no. 2, pp. 96-102, 2013.

[15] G. F. Townsend, J. F. Morgan, S. Tolnai, B. Hazlett, H. J. Morton, and R. W. Shuel, "Studies on the in vitro antitumor activity of fatty acids. I. 10-Hydroxy-2-decenoic acid from royal jelly," Cancer Research, vol. 20, no. 4, pp. 503-510, 1960.

[16] X. Y. Yang, D. S. Yang, J. M. Wang et al., "10-Hydroxy-2-decenoic acid from Royal jelly: a potential medicine for RA," Journal of Ethnopharmacology, vol. 128, no. 2, pp. 314-321, 2010.

[17] H. Izuta, Y. Chikaraishi, M. Shimazawa, S. Mishima, and H. Hara, "10-Hydroxy-2-decenoic acid, a major fatty acid from royal jelly, inhibits VEGF-induced angiogenesis in human umbilical vein endothelial cells," Evidence-Based Complementary and Alternative Medicine, vol. 6, no. 4, pp. 489-494, 2009.

[18] C. J. Kenyon, “The genetics of ageing," Nature, vol. 464, no. 7288, pp. 504-512, 2010.

[19] E. L. Greer and A. Brunet, "Different dietary restriction regimens extend lifespan by both independent and overlapping genetic pathways in C. elegans," Aging Cell, vol. 8, no. 2, pp. 113127, 2009.

[20] S. Brenner, "The genetics of Caenorhabditis elegans," Genetics, vol. 77, no. 1, pp. 71-94, 1974.
[21] D. Garigan, A.-L. Hsu, A. G. Fraser, R. S. Kamath, J. Abringet, and C. Kenyon, "Genetic analysis of tissue aging in Caenorhabditis elegans: a role for heat-shock factor and bacterial proliferation," Genetics, vol. 161, no. 3, pp. 1101-1112, 2002.

[22] R. Hosono, "Sterilization and growth inhibition of Caenorhabditis elegans by 5 -fluorodeoxyuridine," Experimental Gerontology, vol. 13, no. 5, pp. 369-374, 1978.

[23] D. H. Mitchell, J. W. Stiles, J. Santelli, and D. R. Sanadi, "Synchronous growth and aging of Caenorhabditis elegans in the presence of fluorodeoxyuridine," The Journals of Gerontology, vol. 34, no. 1, pp. 28-36, 1979.

[24] B. Lakowski and S. Hekimi, "The genetics of caloric restriction in Caenorhabditis elegans," Proceedings of the National Academy of Sciences of the United States of America, vol. 95, no. 22, pp. 13091-13096, 1998.

[25] D.-H. Kim, D. D. Sarbassov, S. M. Ali et al., "mTOR interacts with raptor to form a nutrient-sensitive complex that signals to the cell growth machinery," Cell, vol. 110, no. 2, pp. 163-175, 2002.

[26] K. Evason, C. Huang, I. Yamben, D. F. Covey, and K. Kornfeld, "Anticonvulsant medications extend worm life-span," Science, vol. 307, no. 5707, pp. 258-262, 2005.

[27] K. Evason, J. J. Collins, C. Huang, S. Hughes, and K. Kornfeld, "Valproic acid extends Caenorhabditis elegans lifespan," Aging Cell, vol. 7, no. 3, pp. 305-317, 2008.

[28] W.-J. Cai, J.-H. Huang, S.-Q. Zhang et al., "Icariin and its derivative icariside II extend healthspan via insulin/IGF-1 pathway in C. elegans," PLoS ONE, vol. 6, no. 12, Article ID e28835, 2011.

[29] S. Havermann, Y. Chovolou, H. U. Humpf, W. Wätjen, and J. Sadoshima, "Caffeic acid phenethylester increases stress resistance and enhances lifespan in Caenorhabditis elegans by modulation of the insulin-like DAF-16 signalling pathway," PLoS ONE, vol. 9, no. 6, Article ID e100256, 2014.

[30] T. J. Schulz, K. Zarse, A. Voigt, N. Urban, M. Birringer, and M. Ristow, "Glucose restriction extends Caenorhabditis elegans life span by inducing mitochondrial respiration and increasing oxidative stress," Cell Metabolism, vol. 6, no. 4, pp. 280-293, 2007.

[31] R. M. Chin, X. Fu, M. Y. Pai et al., "The metabolite $\alpha$ ketoglutarate extends lifespan by inhibiting ATP synthase and TOR," Nature, vol. 510, no. 7505, pp. 397-401, 2014.

[32] A. A. Powolny, S. V. Singh, S. Melov, A. Hubbard, and A. L. Fisher, "The garlic constituent diallyl trisulfide increases the lifespan of C. elegans via skn-1 activation," Experimental Gerontology, vol. 46, no. 6, pp. 441-452, 2011.

[33] B. Onken and M. Driscoll, "Metformin induces a dietary restriction-like state and the oxidative stress response to extend C. elegans healthspan via AMPK, LKB1, and SKN-1," PLoS ONE, vol. 5, no. 1, Article ID e8758, 2010.

[34] F. Cabreiro, C. Au, K.-Y. Leung et al., "Metformin retards aging in C. elegans by altering microbial folate and methionine metabolism," Cell, vol. 153, no. 1, pp. 228-239, 2013.

[35] M. Viswanathan, S. K. Kim, A. Berdichevsky, and L. Guarente, "A role for SIR-2.1 regulation of ER stress response genes in determining C. elegans life span," Developmental Cell, vol. 9, no. 5, pp. 605-615, 2005.

[36] T. M. Bass, D. Weinkove, K. Houthoofd, D. Gems, and L. Partridge, "Effects of resveratrol on lifespan in Drosophila melanogaster and Caenorhabditis elegans," Mechanisms of Ageing and Development, vol. 128, no. 10, pp. 546-552, 2007. 
[37] R. Strong, R. A. Miller, C. M. Astle et al., "Evaluation of resveratrol, green tea extract, curcumin, oxaloacetic acid, and mediumchain triglyceride oil on life span of genetically heterogeneous mice," The Journals of Gerontology Series A: Biological Sciences and Medical Sciences, vol. 68, no. 1, pp. 6-16, 2013.

[38] D. S. Williams, A. Cash, L. Hamadani, and T. Diemer, "Oxaloacetate supplementation increases lifespan in Caenorhabditis elegans through an AMPK/FOXO-dependent pathway," Aging Cell, vol. 8, no. 6, pp. 765-768, 2009.

[39] R. Kucharski, J. Maleszka, S. Foret, and R. Maleszka, "Nutritional control of reproductive status in honeybees via DNA methylation," Science, vol. 319, no. 5871, pp. 1827-1830, 2008.

[40] A. Patel, M. K. Fondrk, O. Kaftanoglu et al., "The making of a queen: TOR pathway is a key player in diphenic caste development," PLoS ONE, vol. 2, no. 6, article e509, 2007.

[41] N. S. Mutti, A. G. Dolezal, F. Wolschin, J. S. Mutti, K. S. Gill, and G. V. Amdam, "IRS and tor nutrient-signaling pathways act via juvenile hormone to influence honey bee caste fate," The Journal of Experimental Biology, vol. 214, no. 23, pp. 3977-3984, 2011.

[42] D. E. Wheeler, N. A. Buck, and J. D. Evans, "Expression of insulin/insulin-like signalling and TOR pathway genes in honey bee caste determination," Insect Molecular Biology, vol. 23, no. 1, pp. 113-121, 2014.

[43] A. Spannhoff, Y. K. Kim, N. J.-M. Raynal et al., "Histone deacetylase inhibitor activity in royal jelly might facilitate caste switching in bees," EMBO Reports, vol. 12, no. 3, pp. 238-243, 2011.

[44] W. X. Wang, L. Q. Tian, Q. Huang, X. B. Wu, and Z. J. Zeng, "Effects of 10-Hydroxy-2-decenoic acid on the development of honey bee (Apis mellifera) larvae," Journal of Apicultural Research, vol. 53, no. 1, pp. 171-176, 2014.

[45] T. Vellai, K. Takacs-Vellai, Y. Zhang, A. L. Kovacs, L. Orosz, and F. Müller, "Influence of TOR kinase on lifespan in C. elegans," Nature, vol. 426, no. 6967, p. 620, 2003.

[46] P. Kapahi, B. M. Zid, T. Harper, D. Koslover, V. Sapin, and S. Benzer, "Regulation of lifespan in Drosophila by modulation of genes in the TOR signaling pathway," Current Biology, vol. 14, no. 10, pp. 885-890, 2004.

[47] P. Kapahi, D. Chen, A. N. Rogers et al., "With TOR, less is more: a key role for the conserved nutrient-sensing TOR pathway in aging," Cell Metabolism, vol. 11, no. 6, pp. 453-465, 2010.

[48] J. J. Wu, J. Liu, E. B. Chen et al., "Increased mammalian lifespan and a segmental and tissue-specific slowing of aging after genetic reduction of mTOR expression," Cell Reports, vol. 4, no. 5, pp. 913-920, 2013.

[49] A. L. Alvers, M. S. Wood, D. Hu, A. C. Kaywell, W. A. Dunn Jr., and J. P. Aris, "Autophagy is required for extension of yeast chronological life span by rapamycin," Autophagy, vol. 5, no. 6, pp. 847-849, 2009.

[50] S. Robida-Stubbs, K. Glover-Cutter, D. W. Lamming et al., “TOR signaling and rapamycin influence longevity by regulating SKN-1/Nrf and DAF-16/FoxO," Cell Metabolism, vol. 15, no. 5, pp. 713-724, 2012.

[51] I. Bjedov, J. M. Toivonen, F. Kerr et al., "Mechanisms of life span extension by rapamycin in the fruit fly Drosophila melanogaster," Cell Metabolism, vol. 11, no. 1, pp. 35-46, 2010.

[52] D. E. Harrison, R. Strong, Z. D. Sharp et al., "Rapamycin fed late in life extends lifespan in genetically heterogeneous mice," Nature, vol. 460, no. 7253, pp. 392-395, 2009.

[53] V. P. Houde, S. Brûlé, W. T. Festuccia et al., "Chronic rapamycin treatment causes glucose intolerance and hyperlipidemia by upregulating hepatic gluconeogenesis and impairing lipid deposition in adipose tissue," Diabetes, vol. 59, no. 6, pp. 1338-1348, 2010.

[54] N. Deblon, L. Bourgoin, C. Veyrat-Durebex et al., "Chronic mTOR inhibition by rapamycin induces muscle insulin resistance despite weight loss in rats," British Journal of Pharmacology, vol. 165, no. 7, pp. 2325-2340, 2012.

[55] D. W. Lamming, L. Ye, P. Katajisto et al., "Rapamycin-induced insulin resistance is mediated by mTORC2 loss and uncoupled from longevity," Science, vol. 335, no. 6076, pp. 1638-1643, 2012.

[56] J. E. Wilkinson, L. Burmeister, S. V. Brooks et al., "Rapamycin slows aging in mice," Aging Cell, vol. 11, no. 4, pp. 675-682, 2012.

[57] F. Neff, D. Flores-Dominguez, D. P. Ryan et al., "Rapamycin extends murine lifespan but has limited effects on aging," The Journal of Clinical Investigation, vol. 123, no. 8, pp. 3272-3291, 2013. 


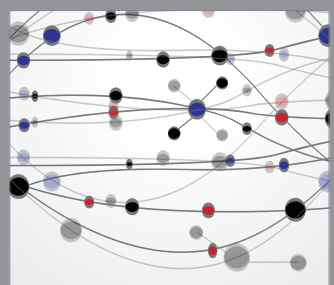

The Scientific World Journal
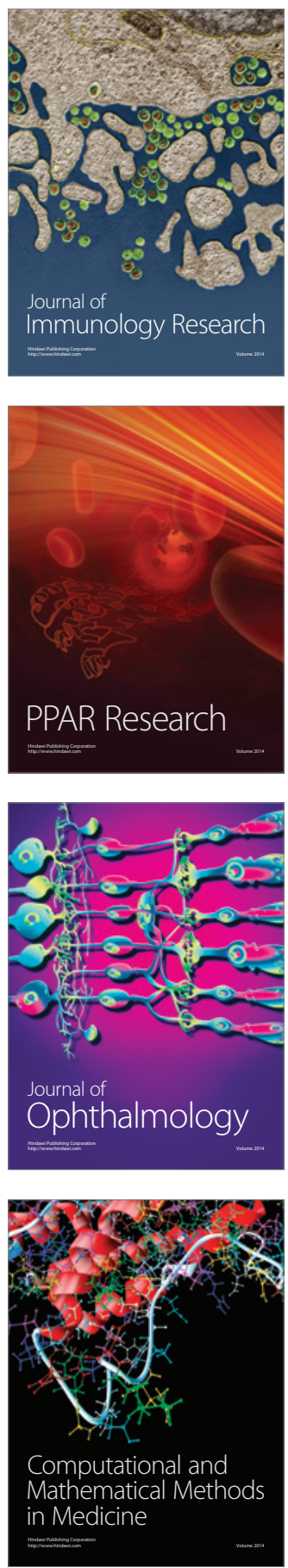

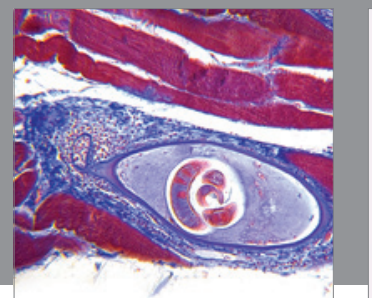

Gastroenterology

Research and Practice
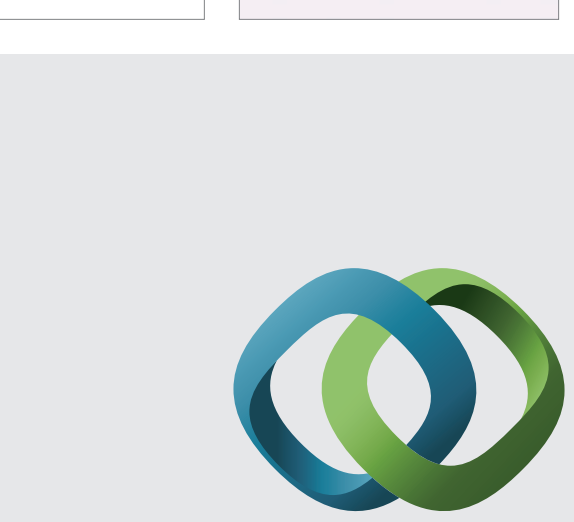

\section{Hindawi}

Submit your manuscripts at

http://www.hindawi.com
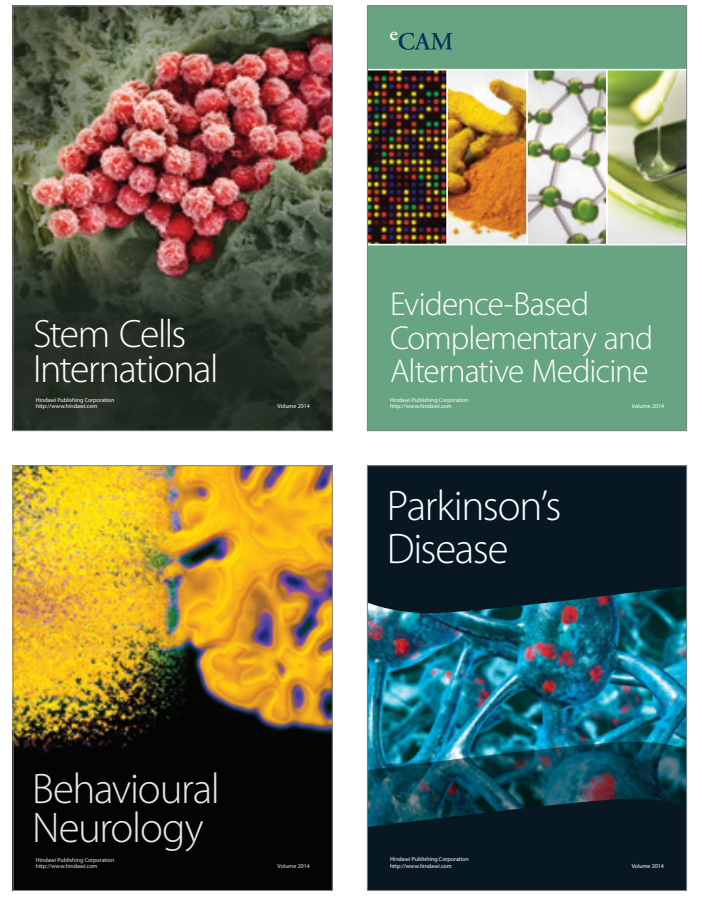
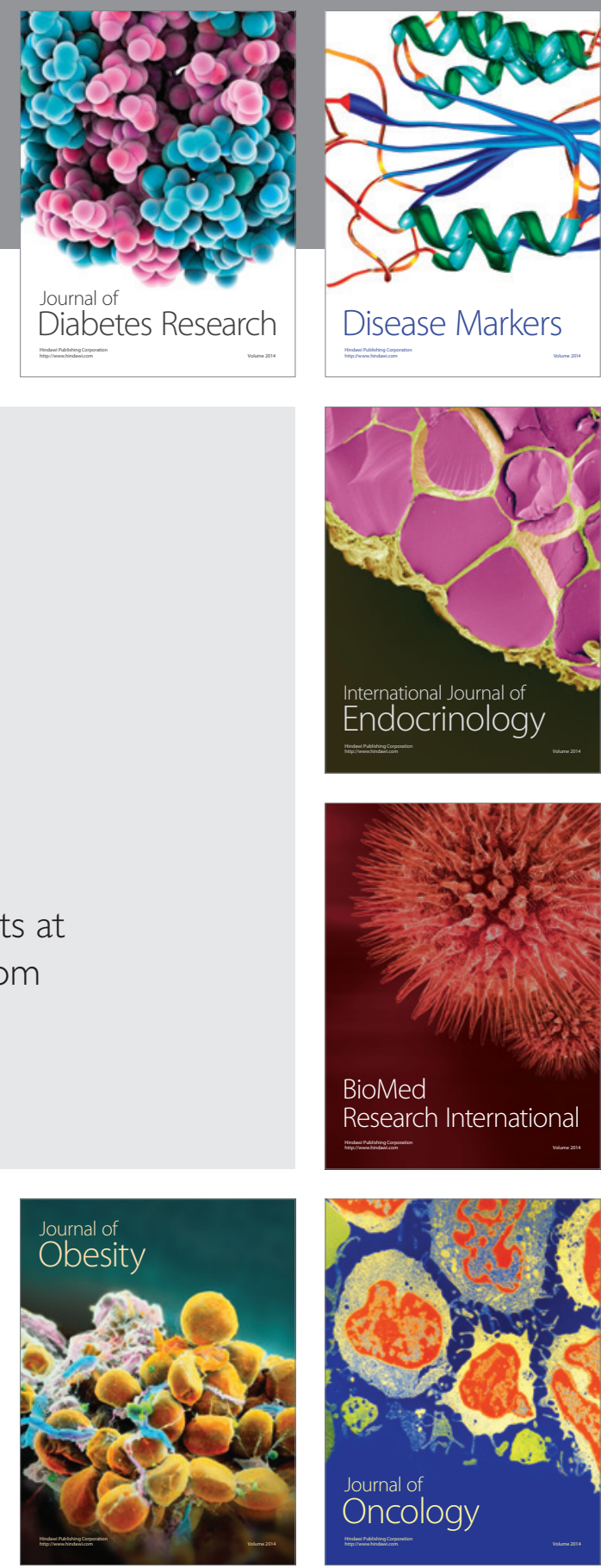

Disease Markers
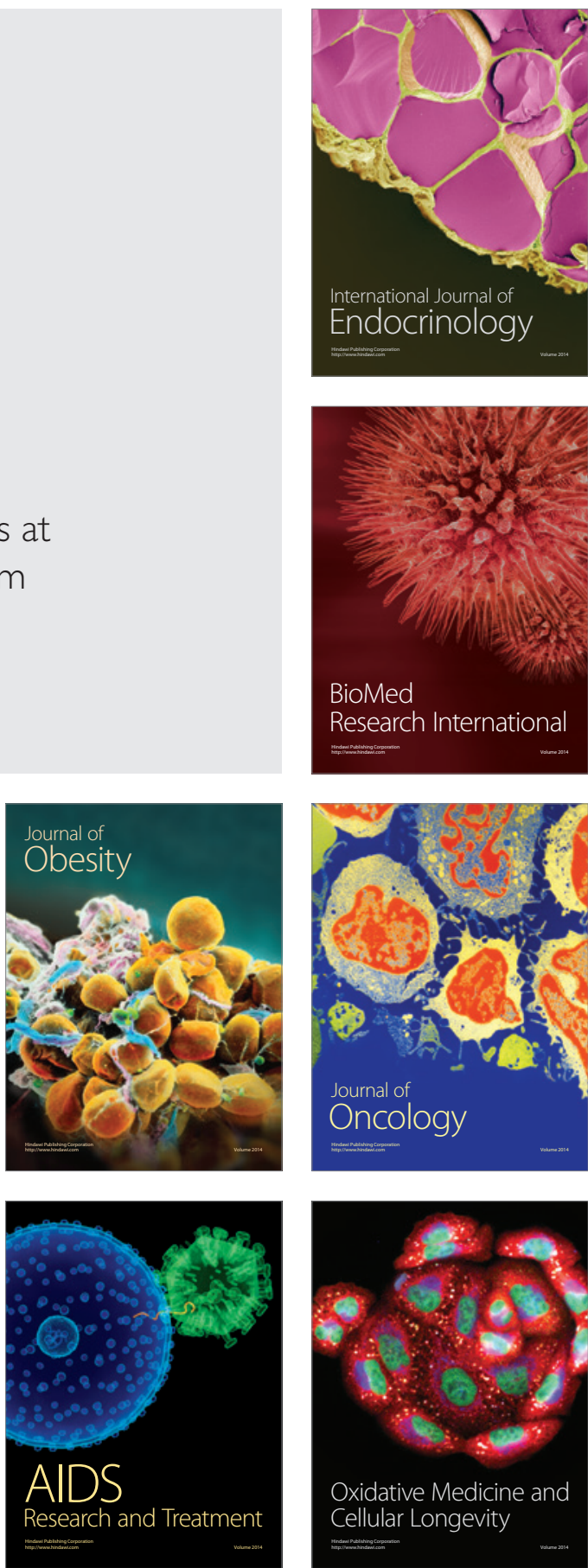